\title{
Early MRI Features of Pediatric Discitis and Spondylodiscitis: An Interactive Quiz With Clinically Challenging Cases.
}

ASNR 2020- Abstract No:

1211

Presenter:

Umber Shafique, MD, CIIP

Neuroradiology Fellow

Department of Radiology and Biomedical Imaging Yale School of Medicine

Dr. William B. Zucconi, DO

Assistant Professor

Neuroradiology Fellowship Director

Department of Radiology and Biomedical Imaging

Yale School of Medicine 


\section{Disclosure:}

- No relevant disclosures. 


\section{Case 1}

- 11 years old female with 3 weeks of abdominal and back pain and one day of nausea and vomiting.

- No fever.

- Borderline elevated WBC's and elevated C-reactive protein.

- Images from her initial MRI are as follows: 

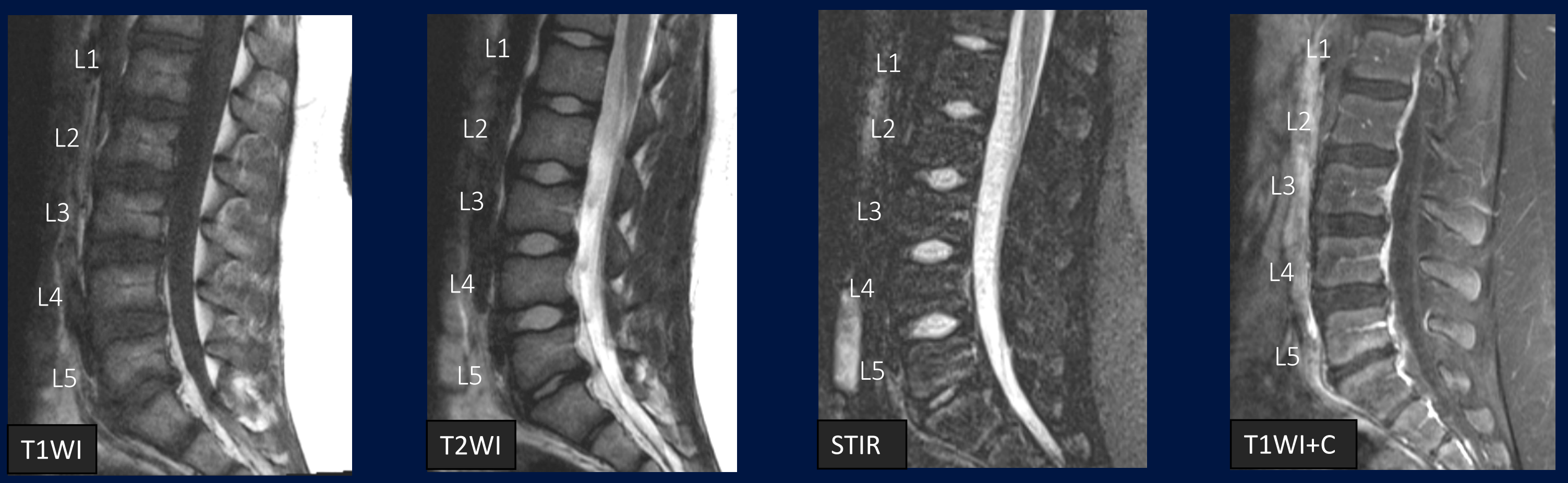

Click on the box to choose all the positive imaging findings.

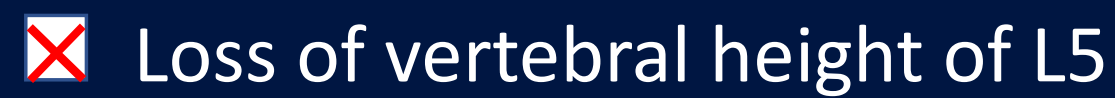

$\checkmark$ Decrease in intervertebral disc height at L5-S1

$\square$ Marrow edema and enhancement at vertebral end plates of L5-S1

$\triangle$ Intervertebral disc enhancement at L5-S1 

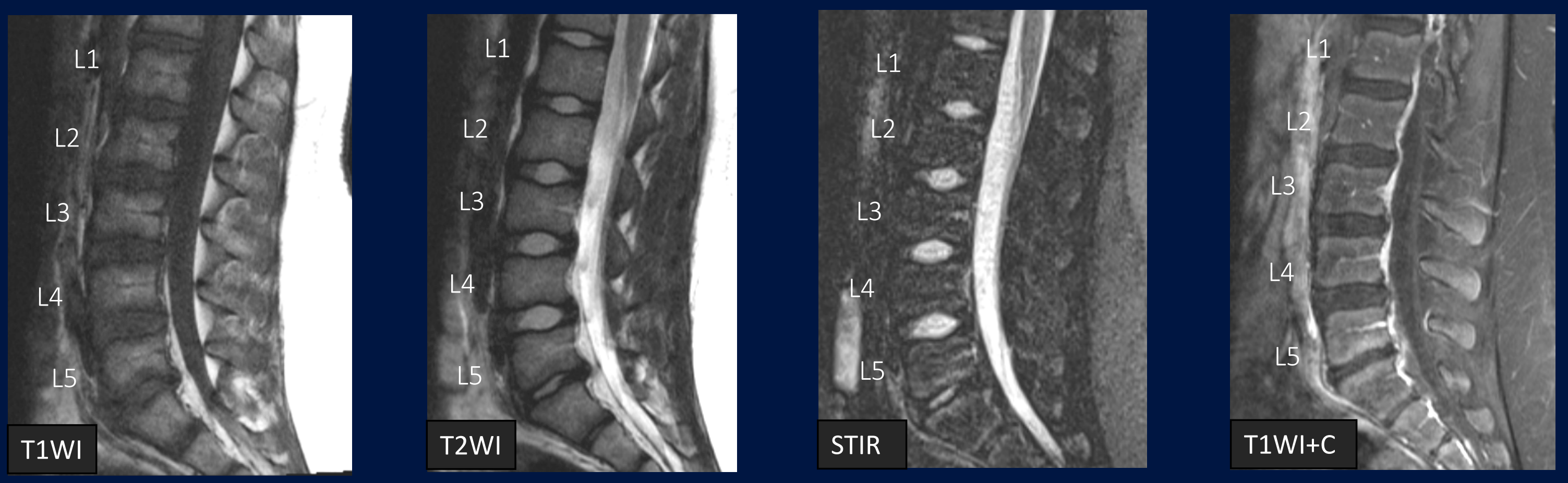

Click on the box to choose all the positive imaging findings.

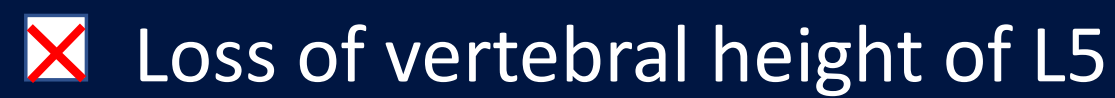

$\checkmark$ Decrease in intervertebral disc height at L5-S1

$\square$ Marrow edema and enhancement at vertebral end plates of L5-S1

$\triangle$ Intervertebral disc enhancement at L5-S1 


\section{Pyogenic discitis}
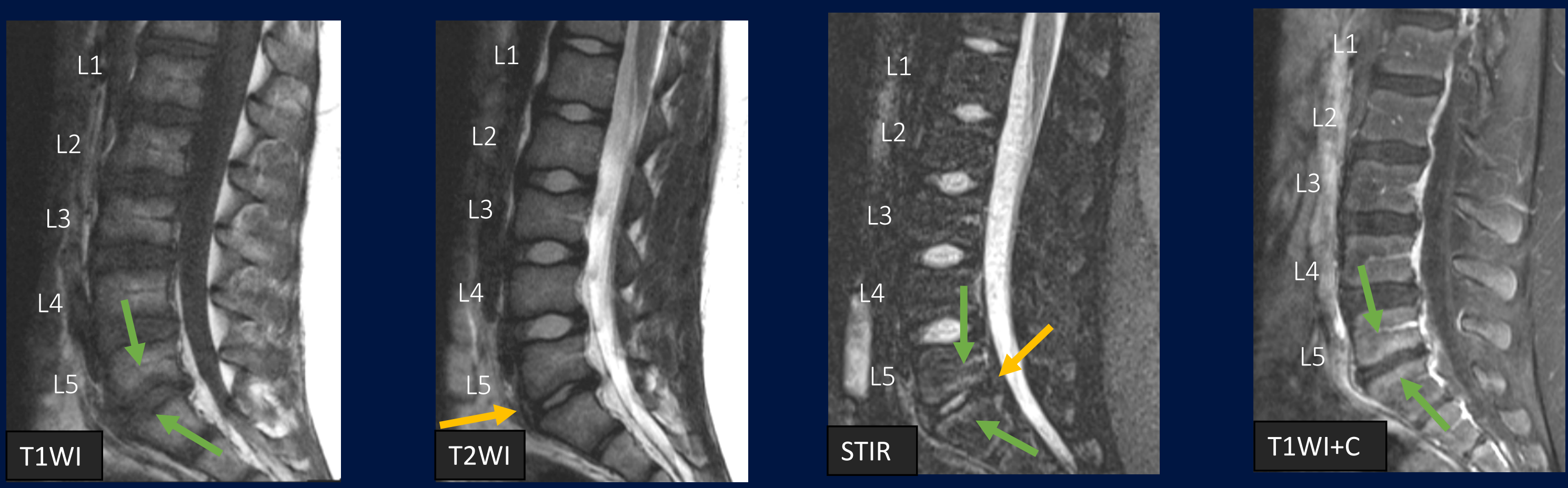

There is loss of intervertebral disc height, mild prevertebral edema with enhancement, and mild marrow changes at vertebral end plates of L5-S1. There is hypointense signal on T1WI, mildly hyperintense signal on STIR with post contrast enhancement at vertebral end plates. No enhancement of intervertebral disc is seen at this time. Findings are suggestive of early discitis. 


\section{Case 1- Continued}

- She is started on broad spectrum intravenous antibiotics and follow up MRI is obtained 2 weeks later. 

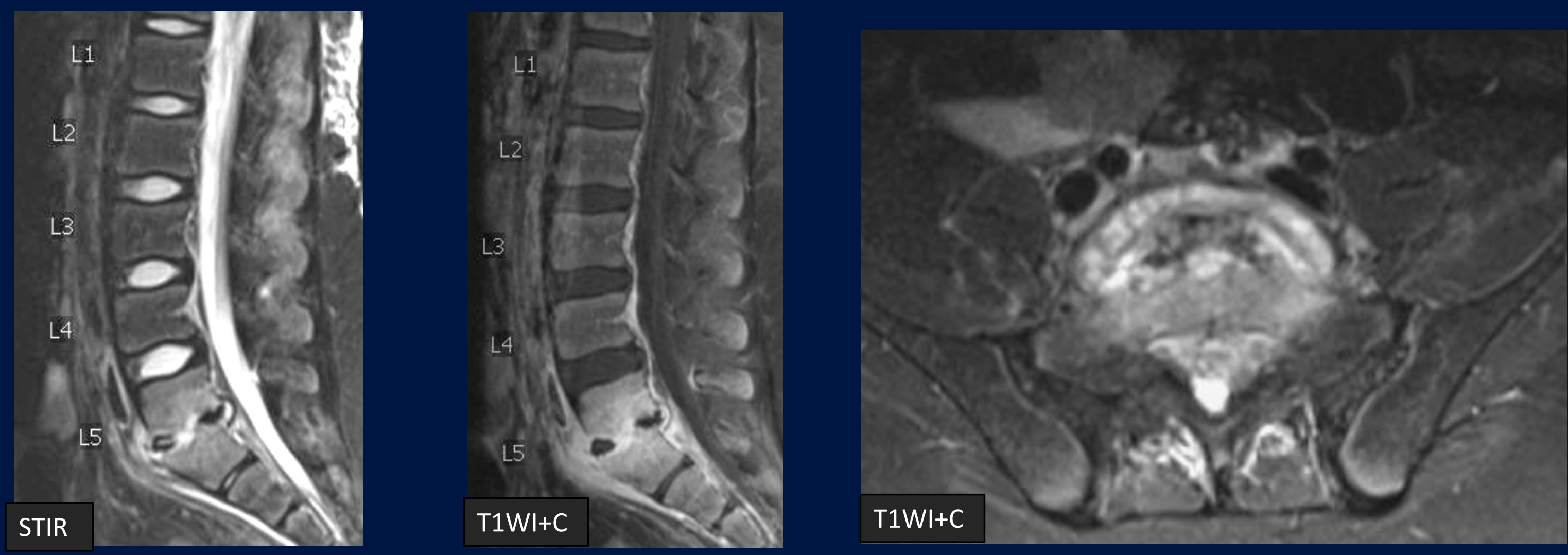

Click on the box to choose all the positive imaging findings.

X Collapse of S1 vertebral body

$\checkmark$ Rim enhancing collection involving anterior intervertebral disc at L5-S1

$\checkmark$ Epidural phlegmon

$\boldsymbol{X}$ Intervertebral disc enhancement at L4-L5 

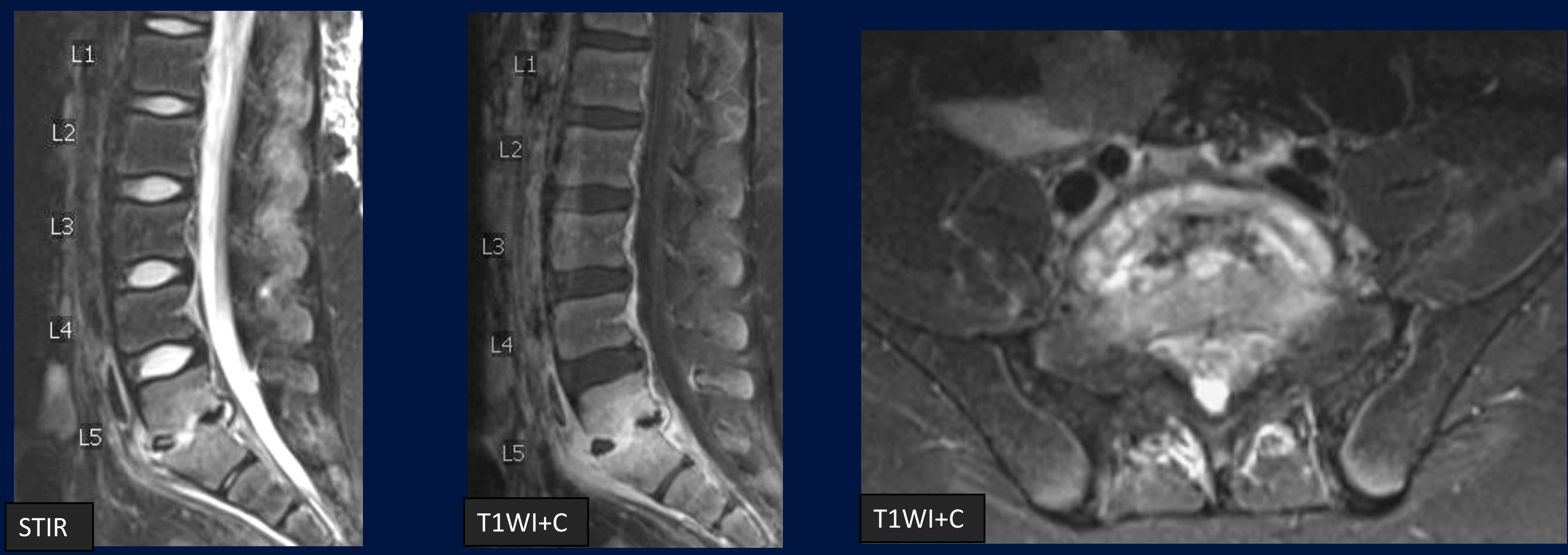

Click on the box to choose all the positive imaging findings.

X Collapse of S1 vertebral body

$\checkmark$ Rim enhancing collection involving anterior intervertebral disc at L5-S1

$\checkmark$ Epidural phlegmon

$\boldsymbol{X}$ Intervertebral disc enhancement at L4-L5 


\section{Pyogenic spondylodiscitis}
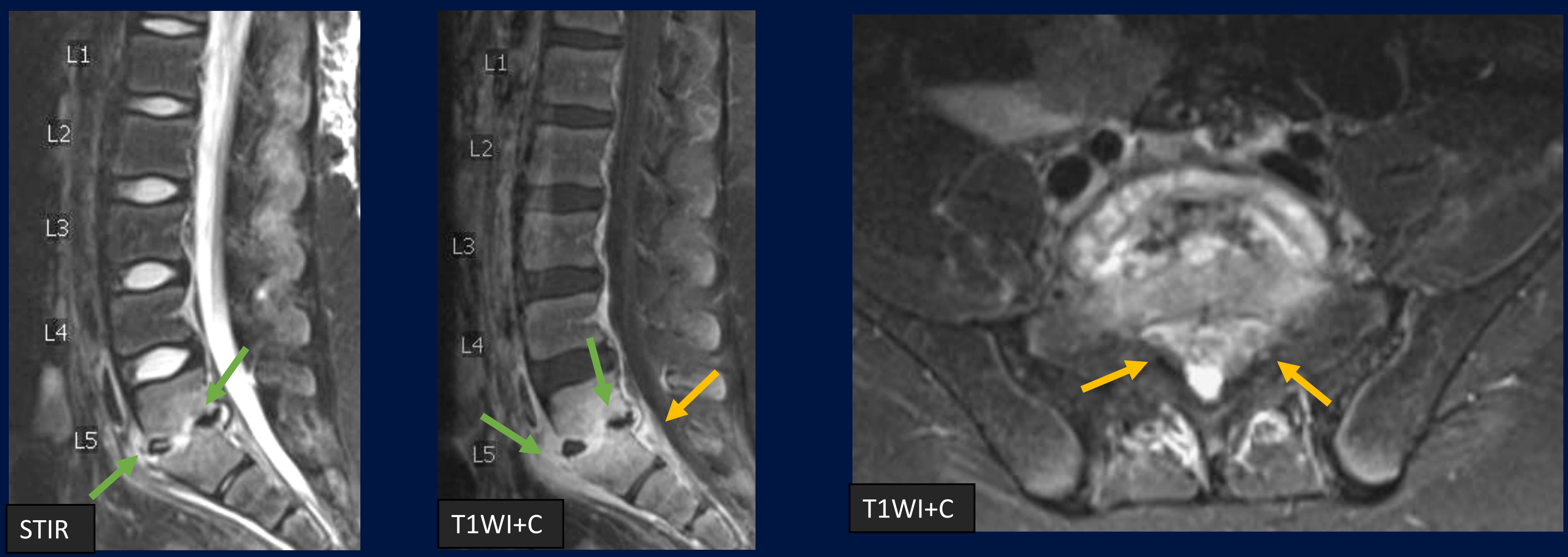

There is complex rim enhancing collection involving intervertebral disc at L5-S1. There is additional epidural phlegmon causing mass effect on ventral thecal sac. Findings are suggestive of spondylodiscitis with intervertebral disc abscess and epidural abscess/phlegmon. The patient underwent CT guided aspiration of intervertebral disc which yielded $5 \mathrm{ml}$ of purulent pus. The culture did not yield growth of an organism. 


\section{Case 2}

- $15 \mathrm{yr}$. old male with back pain following a 5k road race, progressively worsening over 2 weeks, accompanied by "spasms" and right hip pain.

- No fever or elevated WBC's.

- Images from his initial non-contrast MRI are as follows: 


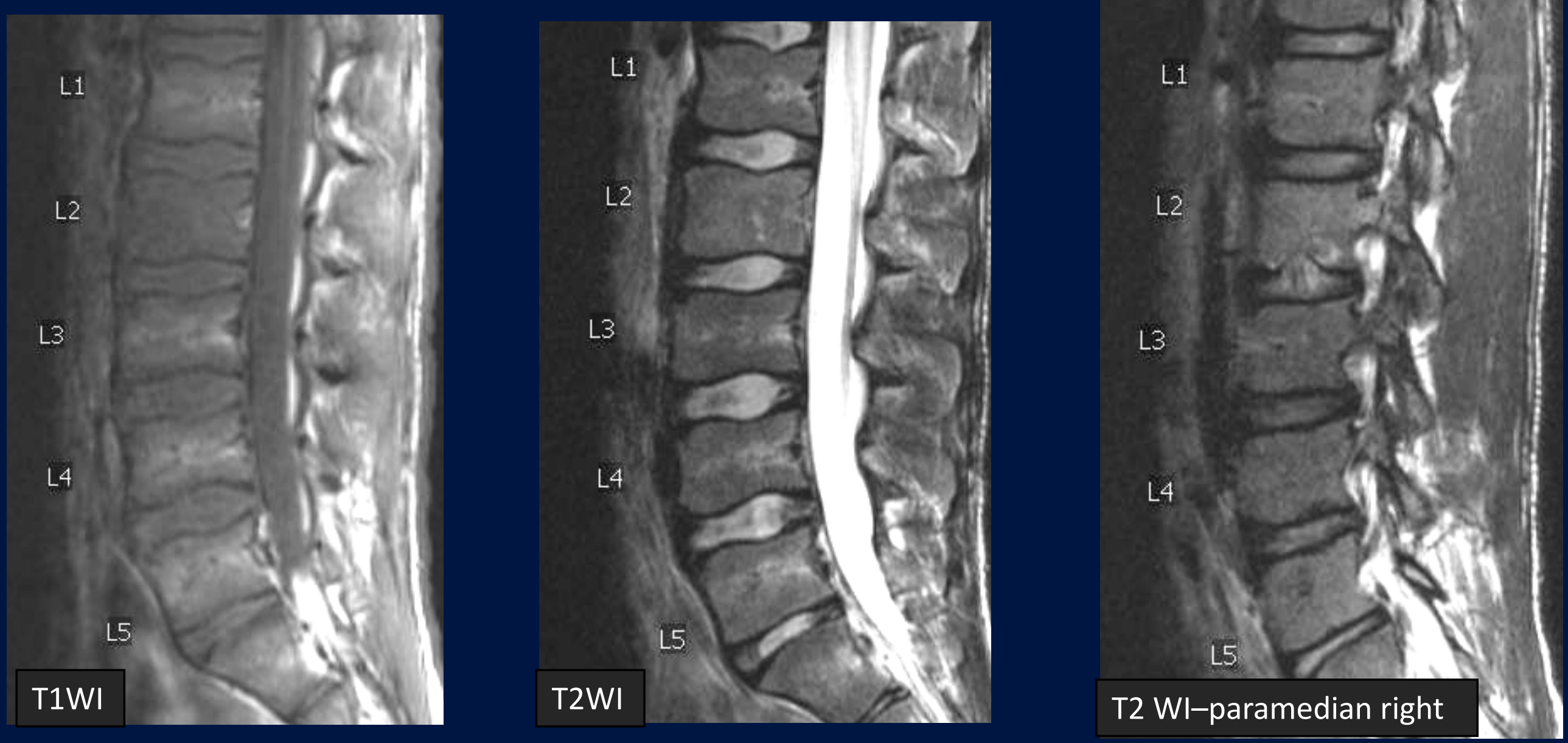

Click on the box to choose all the positive imaging findings.?

\ Loss of vertebral height

$\checkmark$ Hypointense signal on T1WI in L2 vertebral body

X Loss of intervertebral disc height

$\checkmark$ Vertebral end plate defect with superior disc herniation 


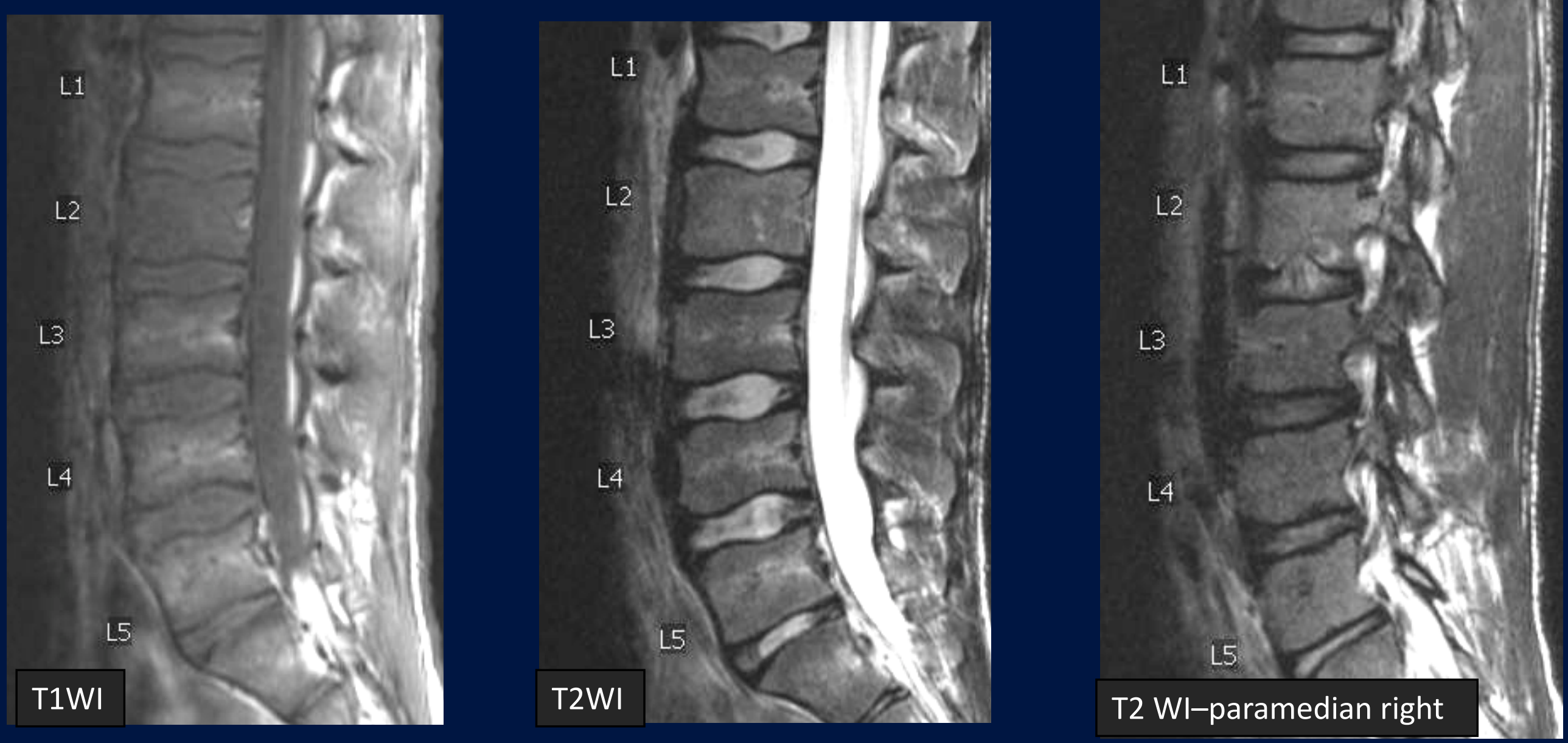

Click on the box to choose all the positive imaging findings.?

$\triangle$ Loss of vertebral height

$\checkmark$ Hypointense signal on T1WI in L2 vertebral body

X Loss of intervertebral disc height

$\checkmark$ Vertebral end plate defect with superior disc herniation 

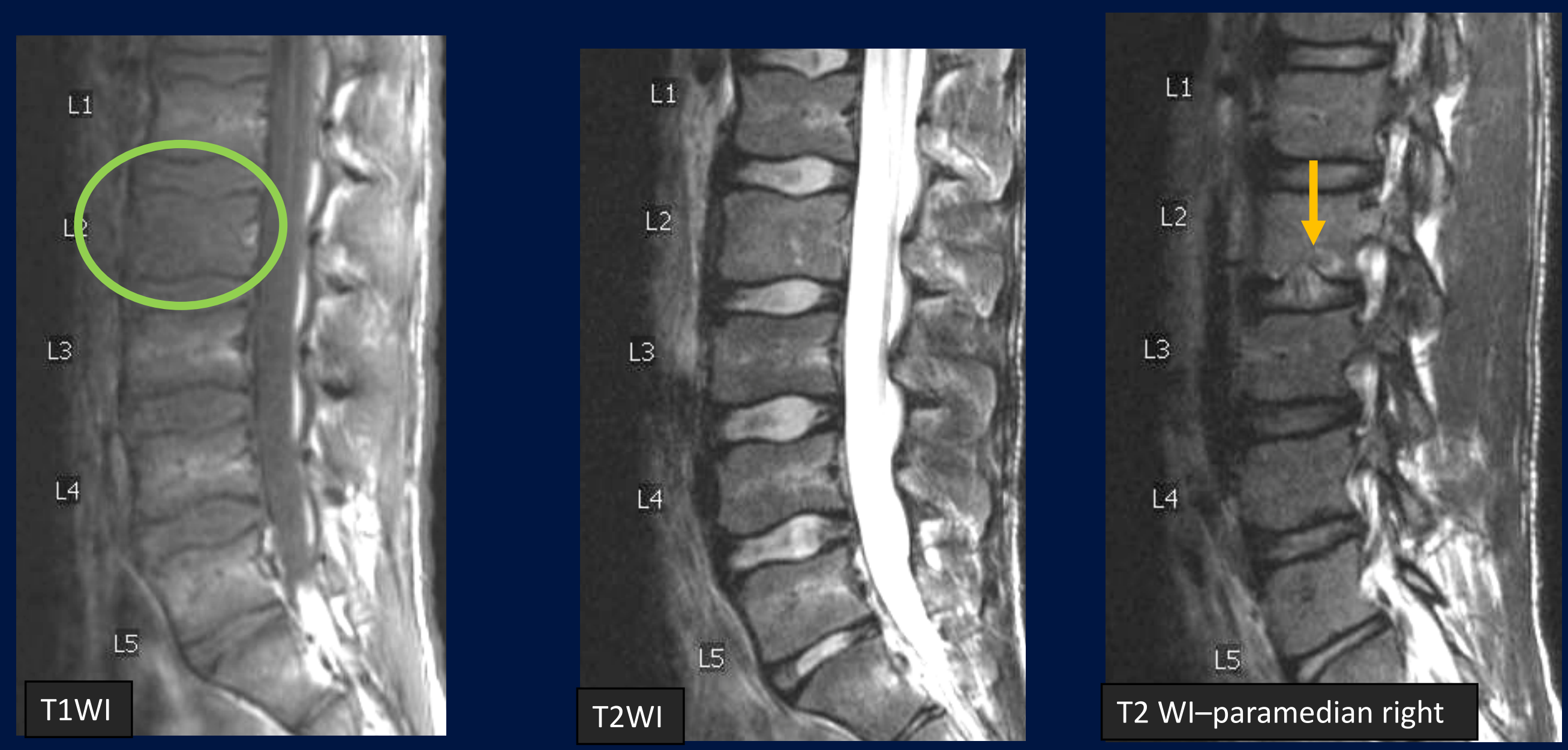

There is hypointense signal on T1WI involving L2 vertebral body. There is vertebral end plate defect with superior disc herniation. Final impression suggested early vertebral spondylitis. 


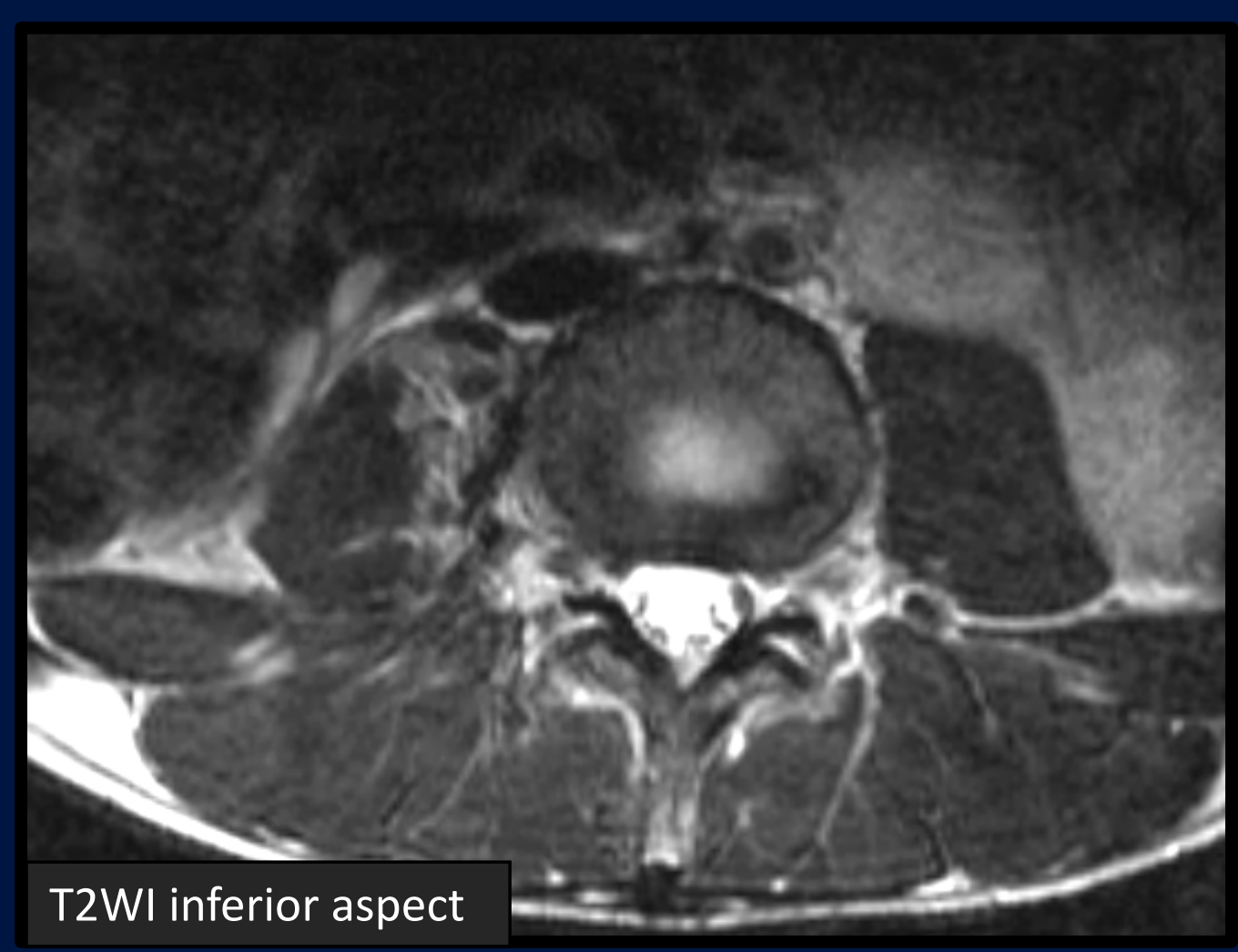

Click on the box to choose all the positive imaging findings.

$\checkmark$ Right paravertebral hyperintense soft tissue

$\triangle$ Left paravertebral hyperintense soft tissue

$X$ Epidural collection

X Spinal canal stenosis 


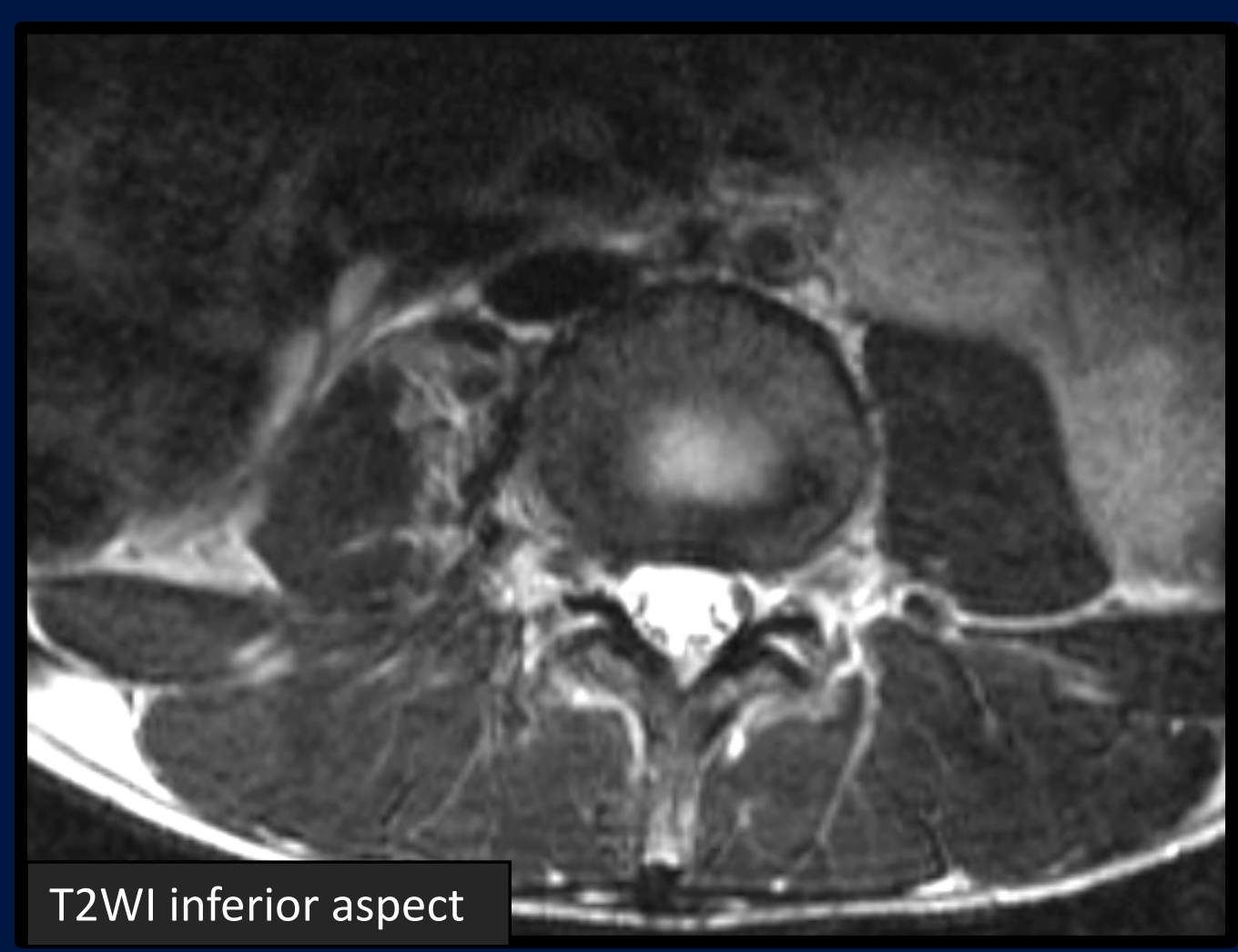

Click on the box to choose all the positive imaging findings.

$\checkmark$ Right paravertebral hyperintense soft tissue

$\triangle$ Left paravertebral hyperintense soft tissue

$X$ Epidural collection

X Spinal canal stenosis 

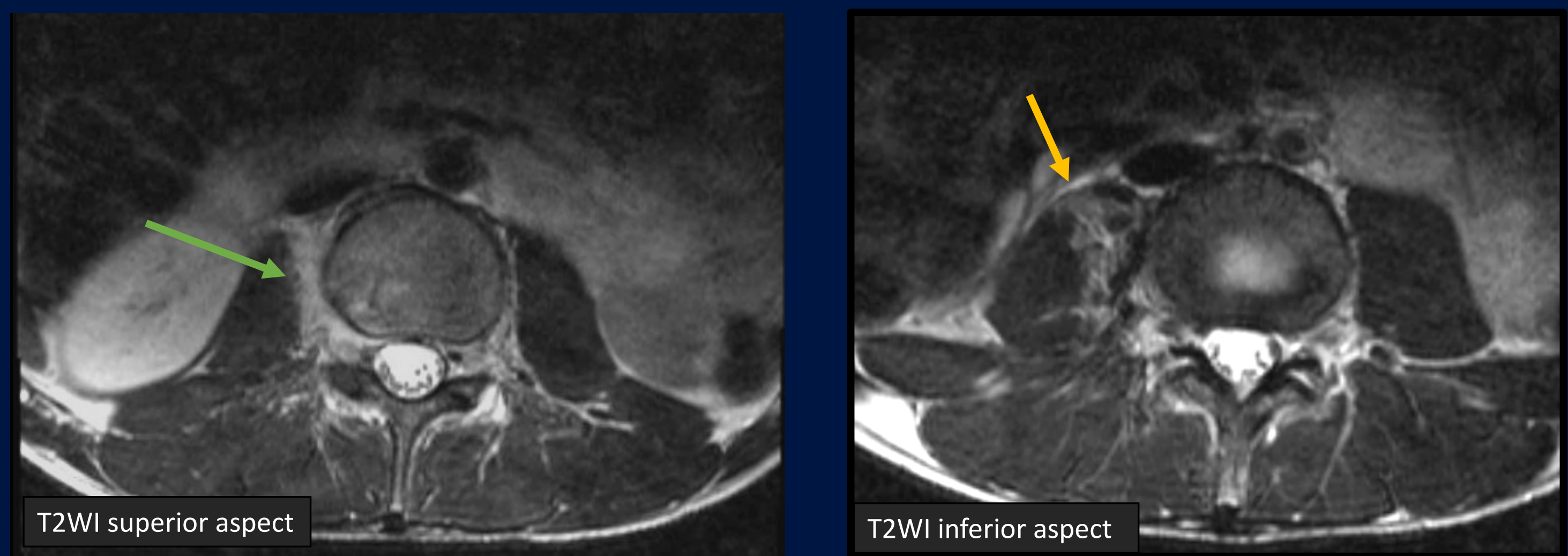

There is asymmetric thickening of right paravertebral soft tissue. It appears to be involving the medial aspect of right psoas muscle inferiorly. Final impression suggested right paraspinal myositis and possible phlegmon. 


\section{Case 2 continued}

- MRI report noted the psoas abnormality and raised the possibility of infection. The patient was treated for muscle strain / injury.

- Over ensuing two weeks, pain worsens, right leg feels "heavy". No numbness or tingling, no bowel or bladder symptoms. ESR and CRP are transiently elevated. The patient is treated with steroids, but symptoms continue to worsen.

- MRI is repeated, 18 days after initial imaging, next slide: 

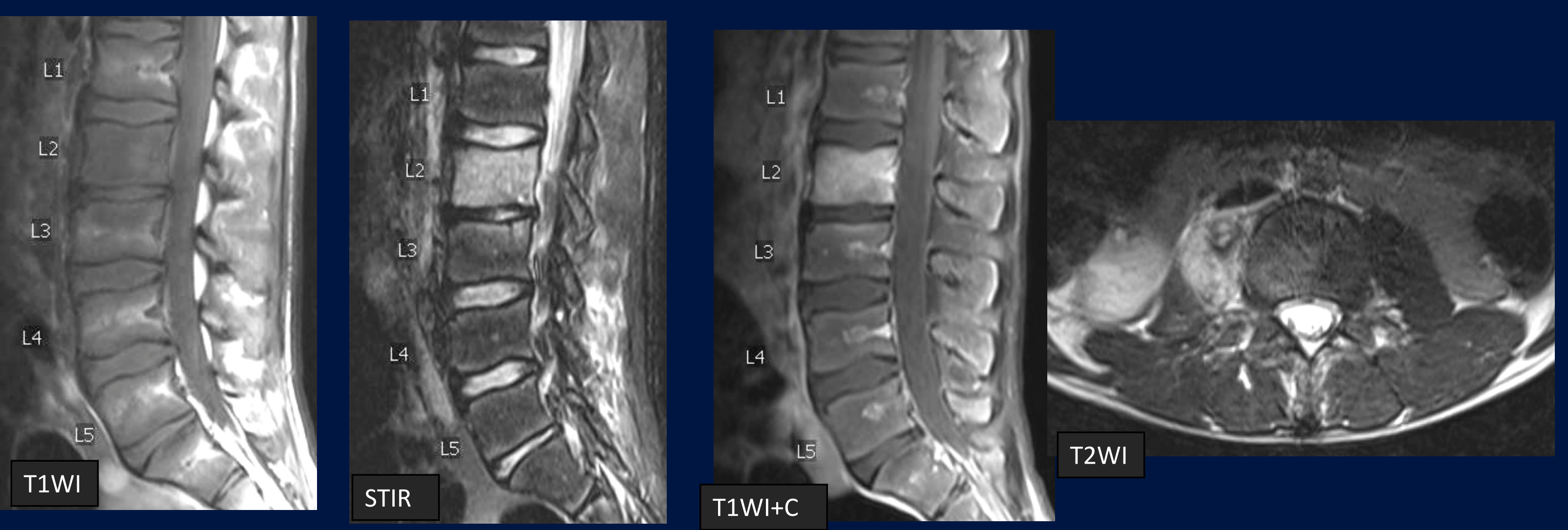

Click on the box to choose all the positive imaging findings.

X Loss of vertebral height

$\checkmark$ Loss of intervertebral disc height at L2-L3 level

$\checkmark$ Phlegmon in right psoas

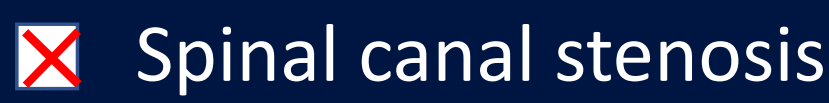



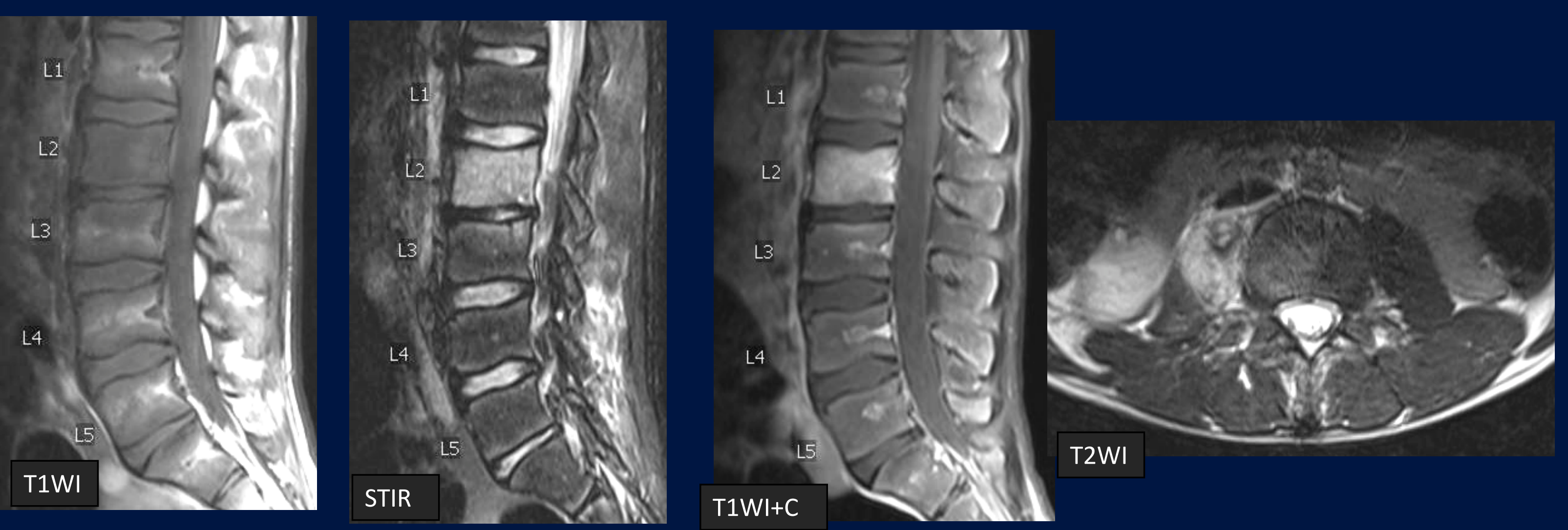

Click on the box to choose all the positive imaging findings.

X Loss of vertebral height

$\checkmark$ Loss of intervertebral disc height at L2-L3 level

$\checkmark$ Phlegmon in right psoas

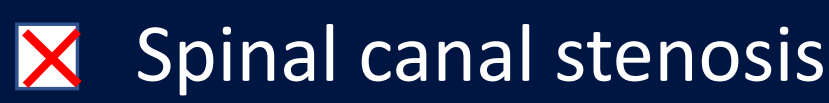




\section{Pyogenic spondylodiscitis}
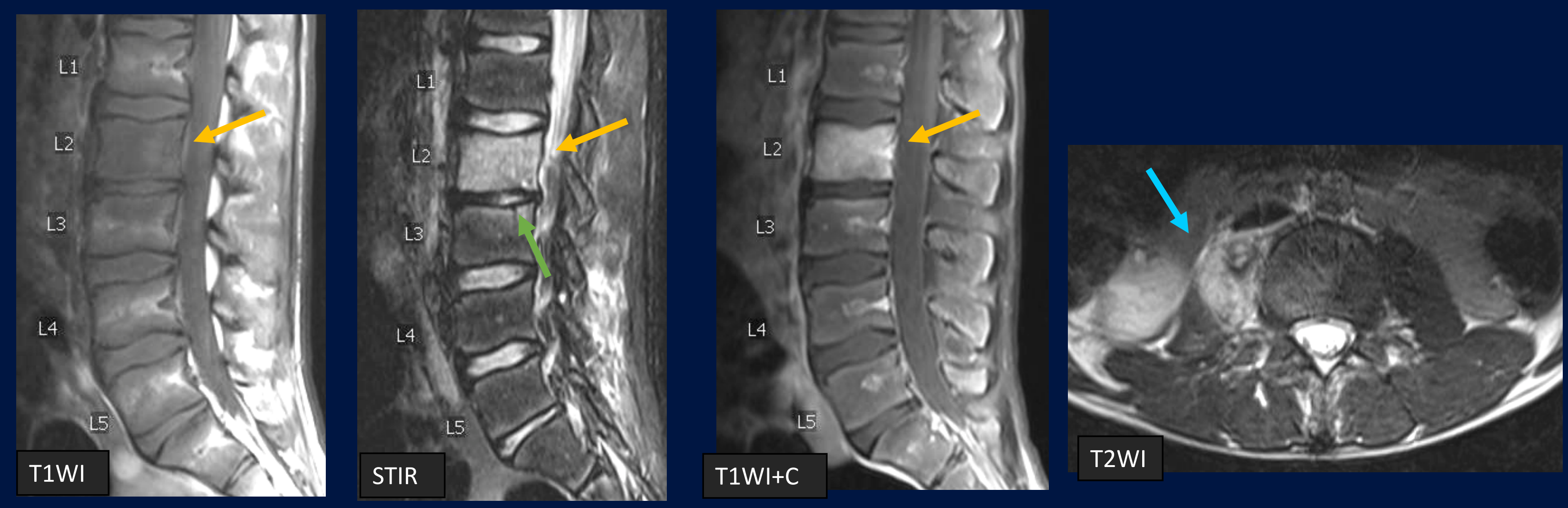

Progression of spondylitis of L2 vertebral body to discitis at L2-L3 intervertebral level. It is seen as hyperintense signal on STIR with post contrast enhancement. There is decrease in intervertebral disc height consistent with early discitis. The right psoas muscle shows infectious myositis / phlegmon. 


\section{Case 3}

- 11 yrs old M who underwent tonsillectomy and adenoidectomy followed by severe post-operative neck pain and stiffness for several weeks.

- There was no fever, elevated WBC or CRP. Initial MRI is as follows: 

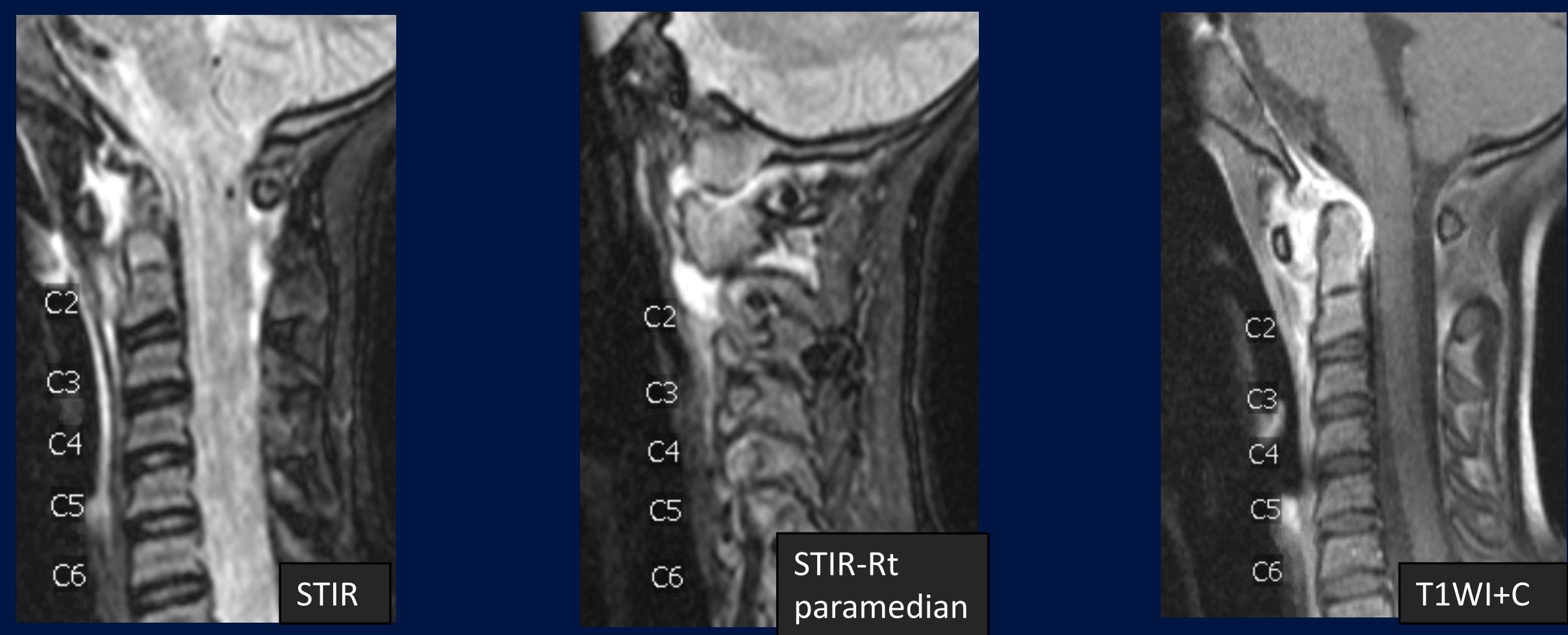

Click on the box to choose all the positive imaging findings.

$\checkmark$ Increase in atlantodental interval

$\checkmark$ Hyperintense signal in right O-C1 joint and C1-C2 facet joint

X Superior migration of dens

$\checkmark$ Enhancing soft tissue surrounding dens 

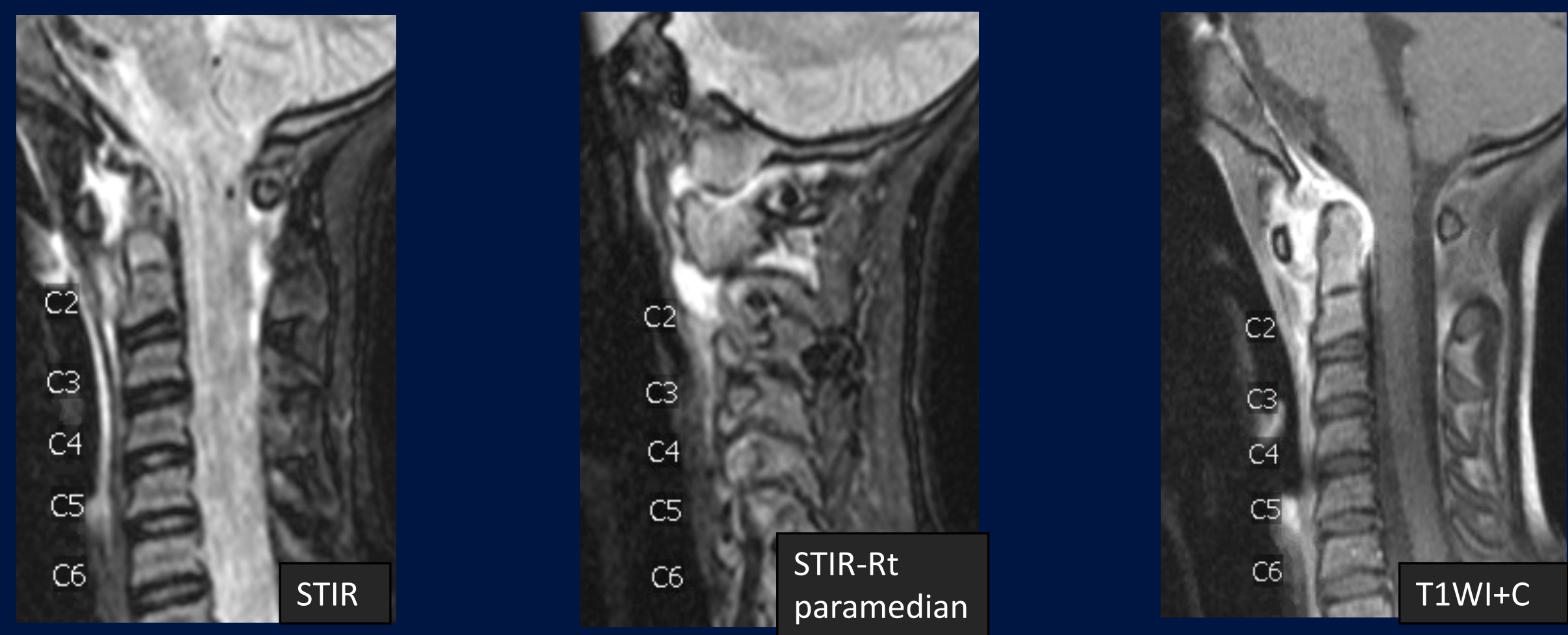

Click on the box to choose all the positive imaging findings.

$\checkmark$ Increase in atlantodental interval

$\checkmark$ Hyperintense signal in right O-C1 joint and C1-C2 facet joint

X Superior migration of dens

$\checkmark$ Enhancing soft tissue surrounding dens 


\section{Right 01-C1 and C1-C2 septic arthritis}
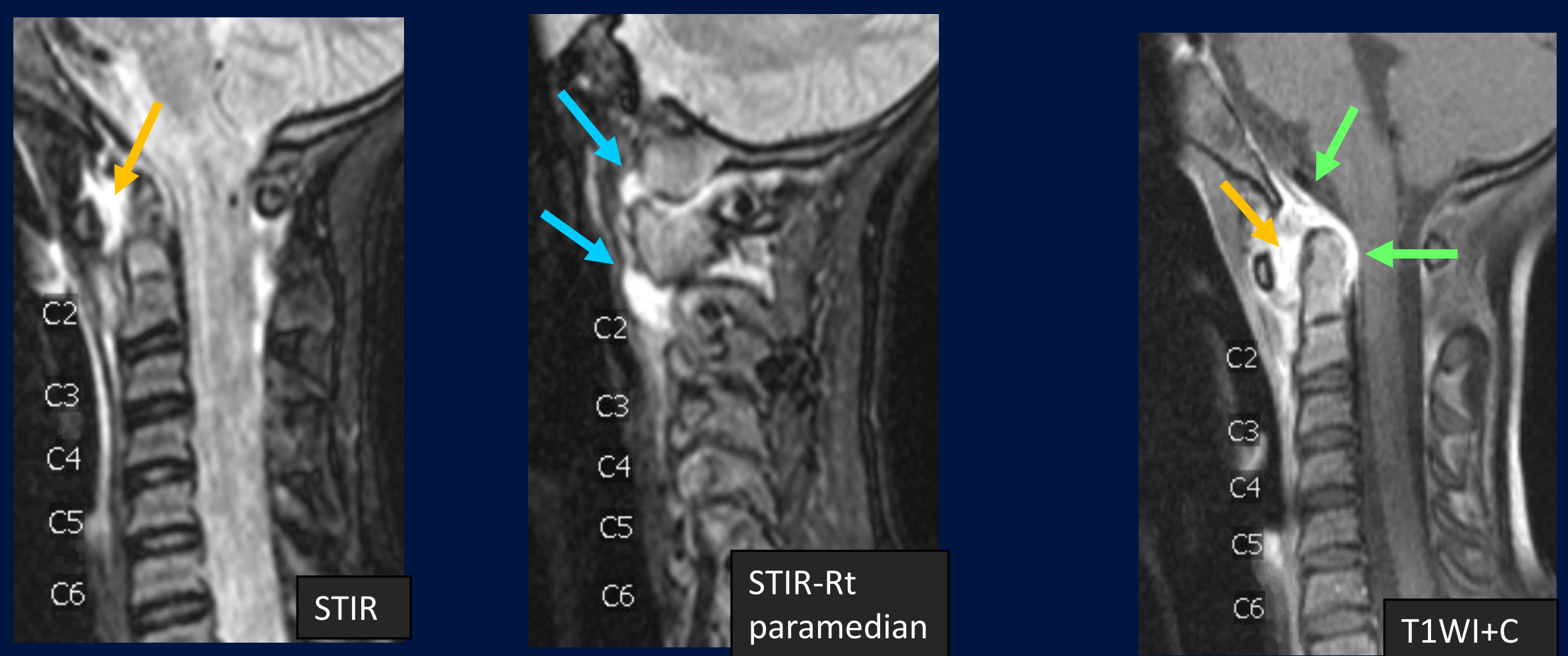

There is soft tissue surrounding the dens which is hyperintense on STIR and shows post contrast enhancement. There is also increase in atlantodental interval. There is also effusion and synovitis in right occipito-atlantic joint and atlanto-axial joint. 

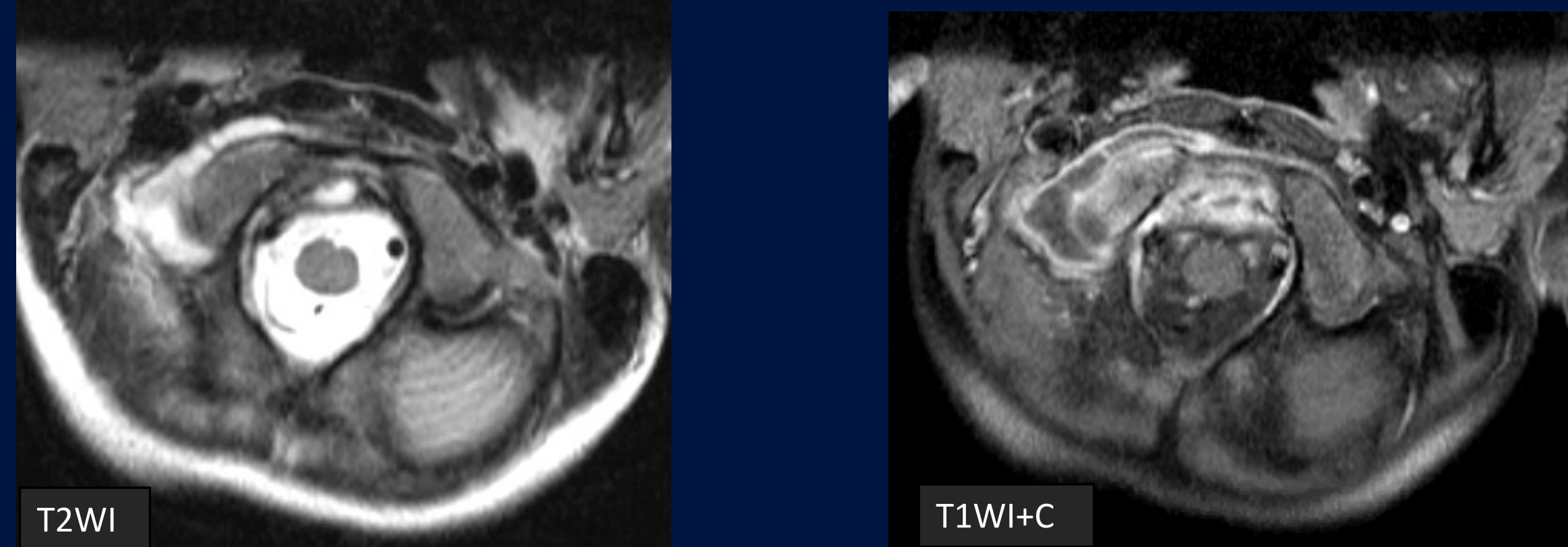

Click on the box to choose all the positive imaging findings.

X Fluid in left C1- C2 facet joint

$\checkmark$ Ventral epidural enhancement and fluid.

$\checkmark$ Rim enhancing collection in right $\mathrm{C} 1-\mathrm{C} 2$ facet joint

$\triangle$ Rim enhancing collection in left C1-C2 facet joint 

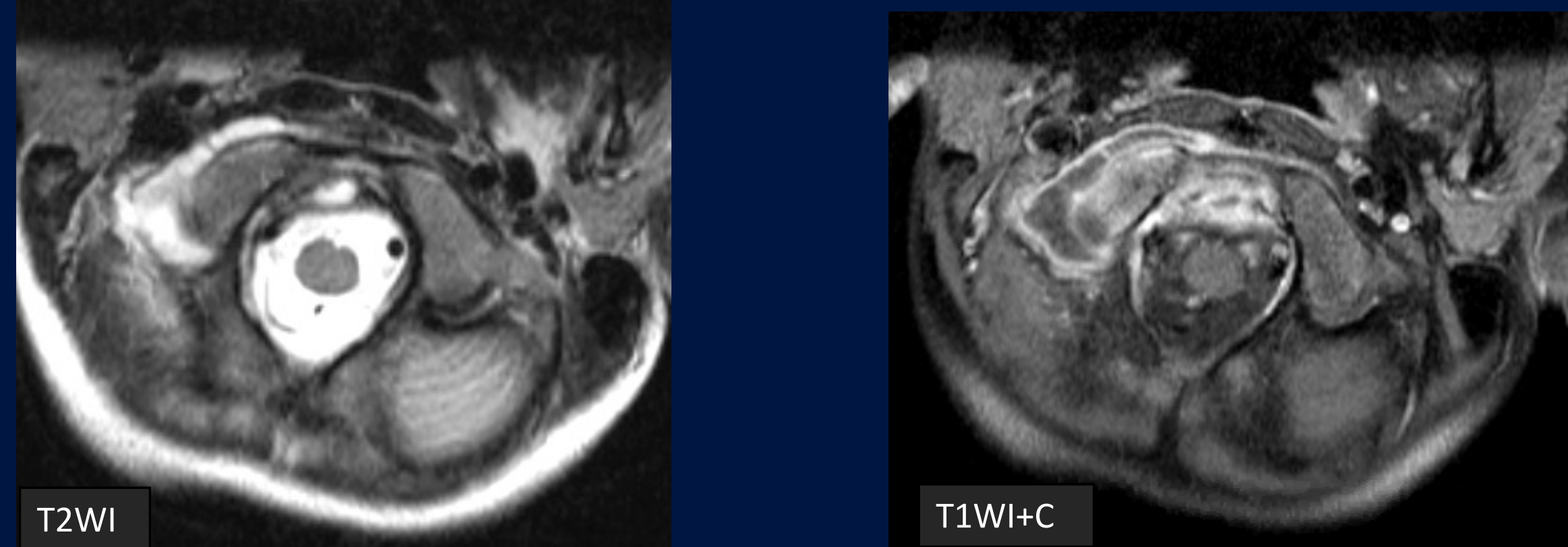

Click on the box to choose all the positive imaging findings.

X Fluid in left C1- C2 facet joint

$\checkmark$ Ventral epidural enhancement and fluid

$\checkmark$ Rim enhancing collection in right $\mathrm{C} 1-\mathrm{C} 2$ facet joint

$\triangle$ Rim enhancing collection in left C1-C2 facet joint 


\section{Right 01-C1 and C1-C2 septic arthritis}
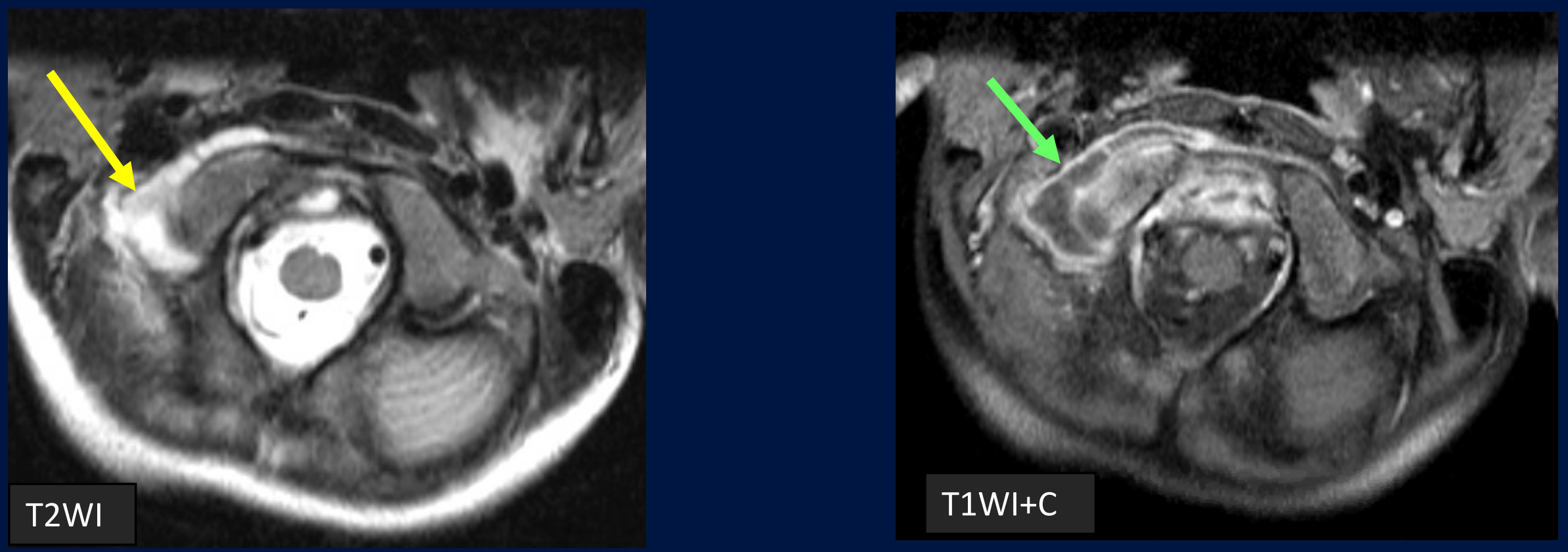

There is prominent joint effusion and fluid collection on T2WI which shows peripheral rim enhancement in right C1-C2 facet joint . Final impression suggested right C1-C2 septic arthritis. 


\section{Case 3 continued}

- Patient was started on IV antibiotics. Follow up MRI in 5 days is as follows: 

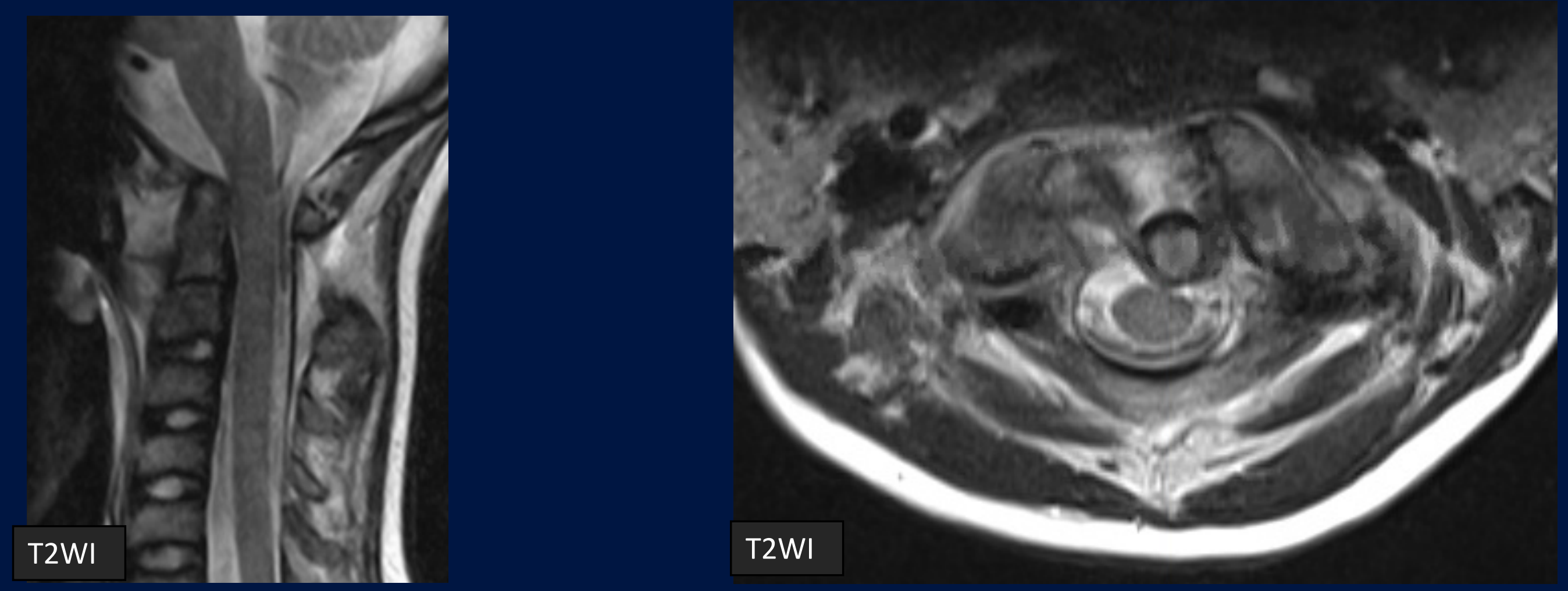

Click on the box to choose all the positive imaging findings.

$\checkmark$ Increase in atlantodental interval

$\triangle$ Decrease in atlantodental interval

$\checkmark$ Craniocervical spinal canal stenosis

$\checkmark$ Atlantotaxial lateral subluxation 

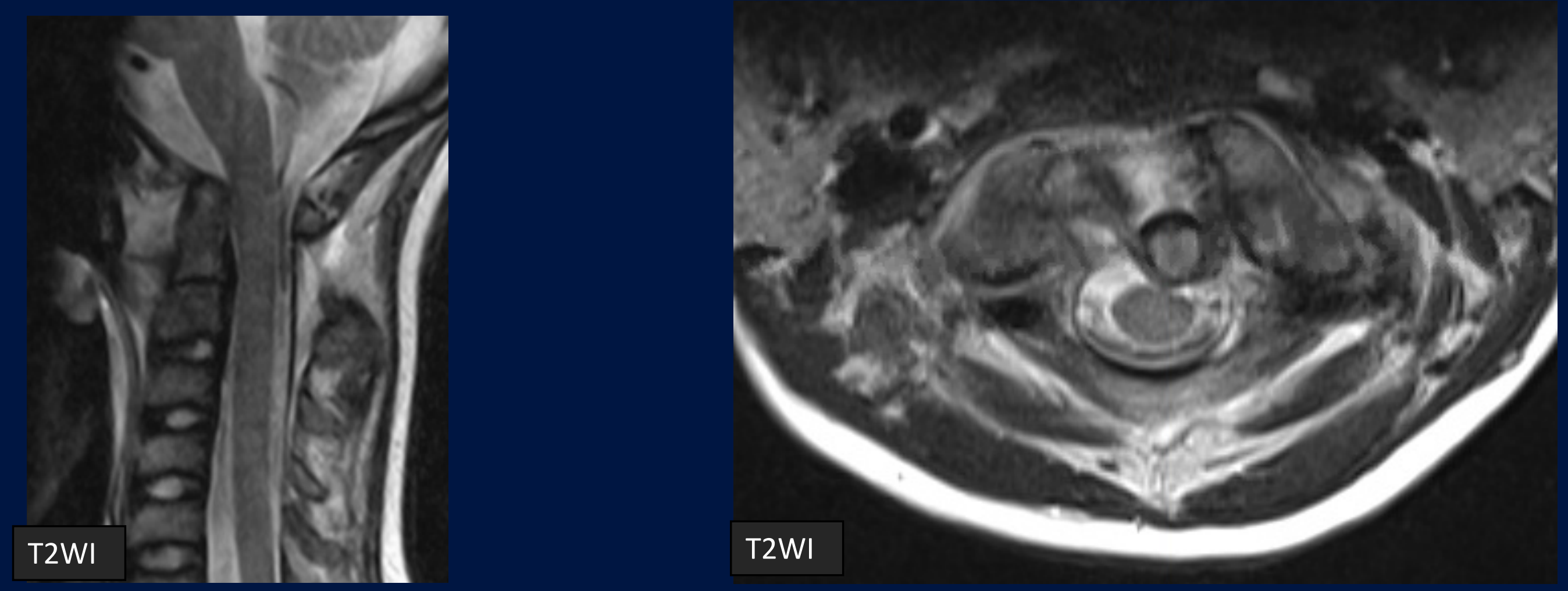

Click on the box to choose all the positive imaging findings.

$\checkmark$ Increase in atlantodental interval

$\triangle$ Decrease in atlantodental interval

$\checkmark$ Craniocervical spinal canal stenosis

$\checkmark$ Atlantotaxial lateral subluxation 


\section{Right $01-\mathrm{C} 1$ and $\mathrm{C} 1-\mathrm{C} 2$ septic arthritis with Grisel syndrome}
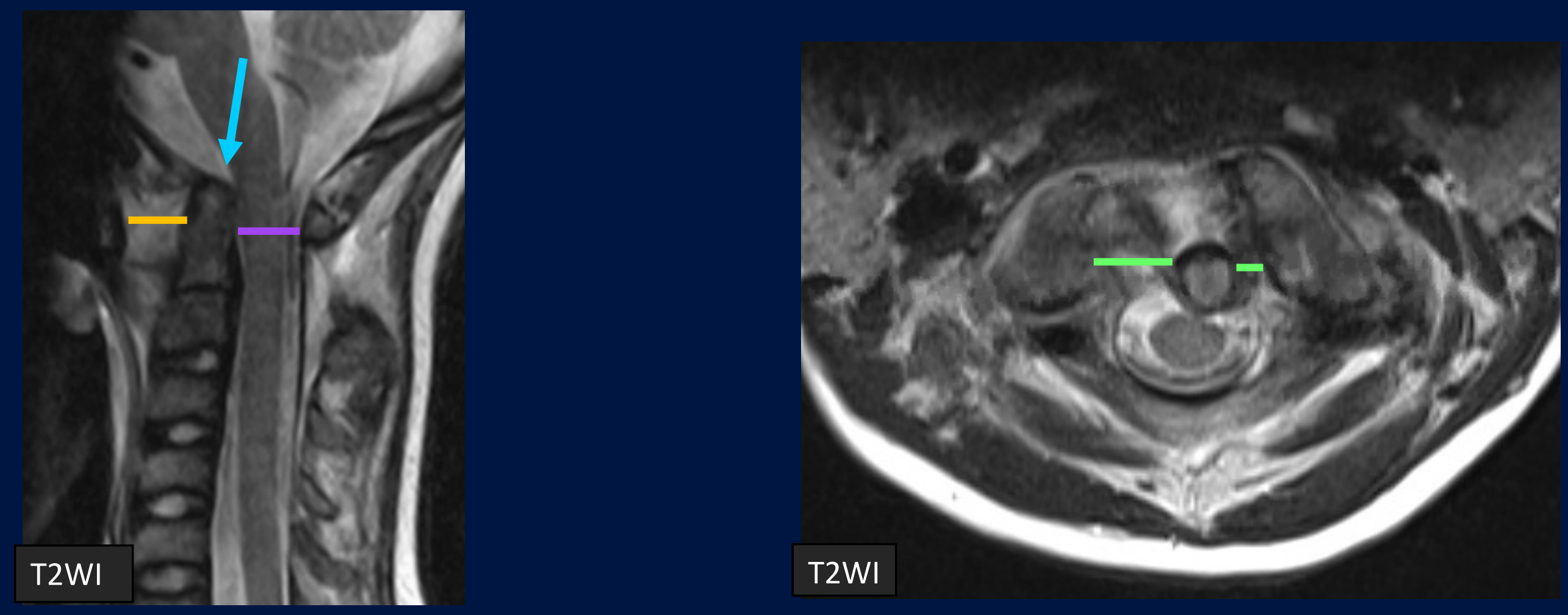

There is now increase in atlantodental interval (normal is less than $5 \mathrm{~mm}$ in children). There is cranial migration of odontoid. There is decrease in spinal canal diameter at craniocervical junction. There is leftwards displacement of odontoid suggestive of lateral subluxation, which suggests Grisel syndrome. 


\section{Right $\mathrm{O} 1-\mathrm{C} 1$ and $\mathrm{C} 1-\mathrm{C} 2$ septic arthritis with Grisel syndrome}

\section{Before: Normal} craniocervical junction with tip of dens well below the McRae line

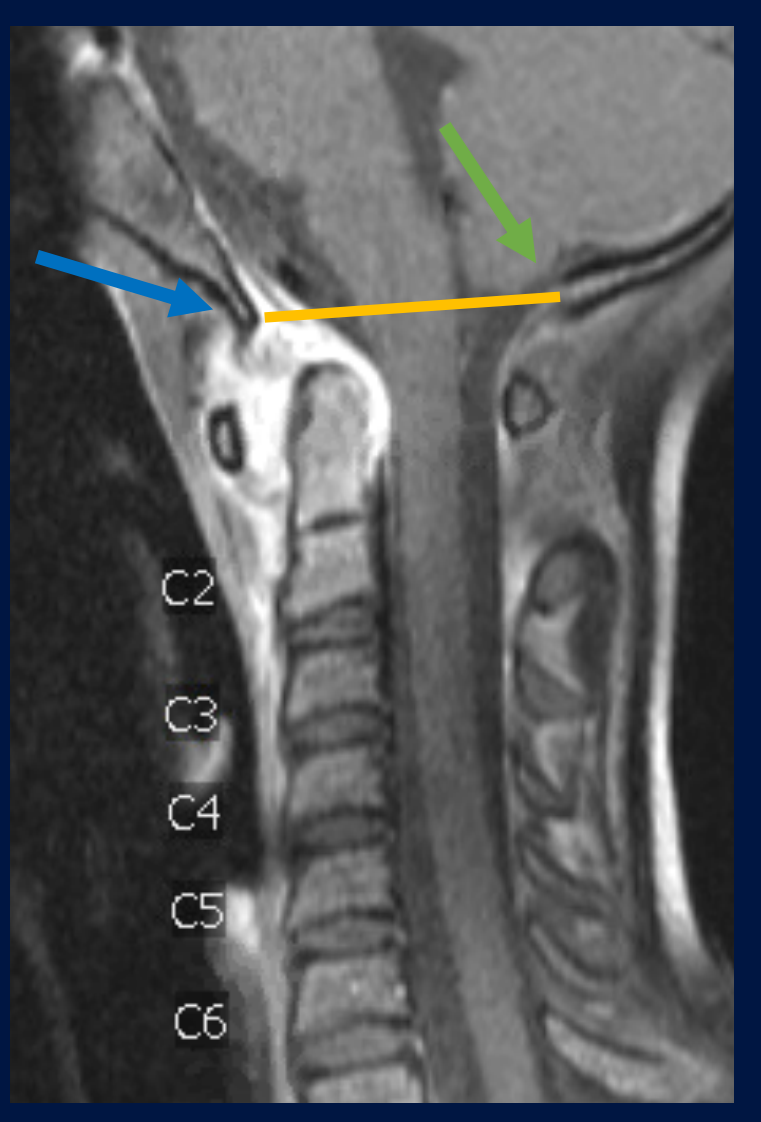

After: Superior migration of tip of dens now closer to the McRae line nearing basilar impression.

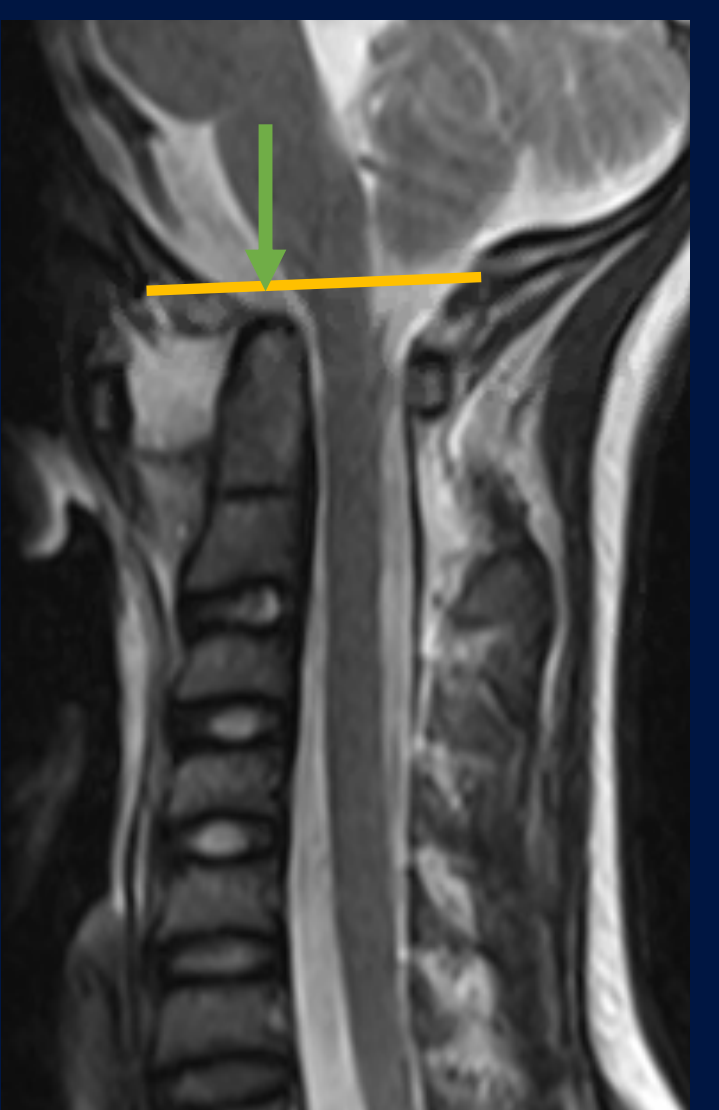

McRae line. Mc Rae line is drawn in midline, between basion and opisthion which are the anterior and posterior margins of foramen magnum, respectively. Basilar impression is the phenomenon when there is relative upward migration of cervical complex/ C2 into the cranial vault secondary to acquired causes. In this case, it is caused by C1-C2 inflammation/ infection. In comparison, Basilar invagination is developmental form of prolapse of the odontoid into the foramen magnum for e.g. Congenital O-A fusion, Klippel-Feil etc, Chiari I. 


\section{Right 01-C1 and C1-C2 septic arthritis with Grisel syndrome}

Before: The dens is equidistant from lateral mass of $\mathrm{C} 1$ which is normal.

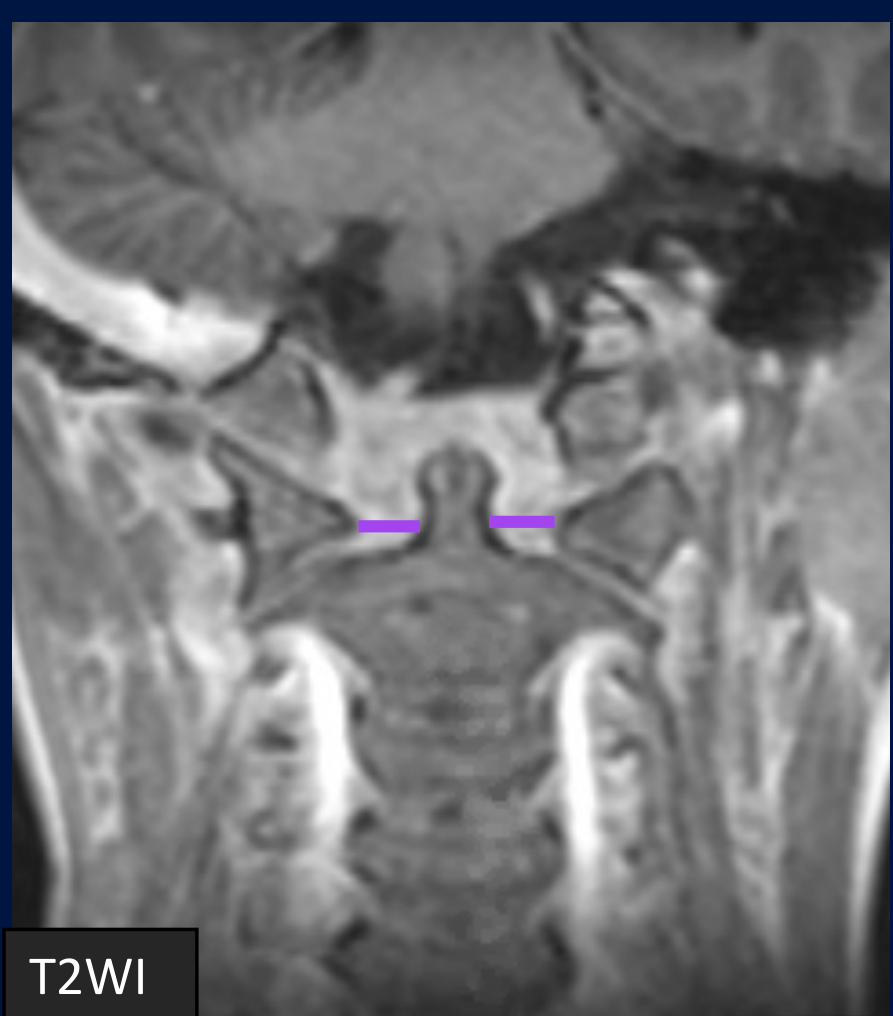

After: There is leftward

displacement of odontoid process with decrease distance between the dens and left lateral mass.

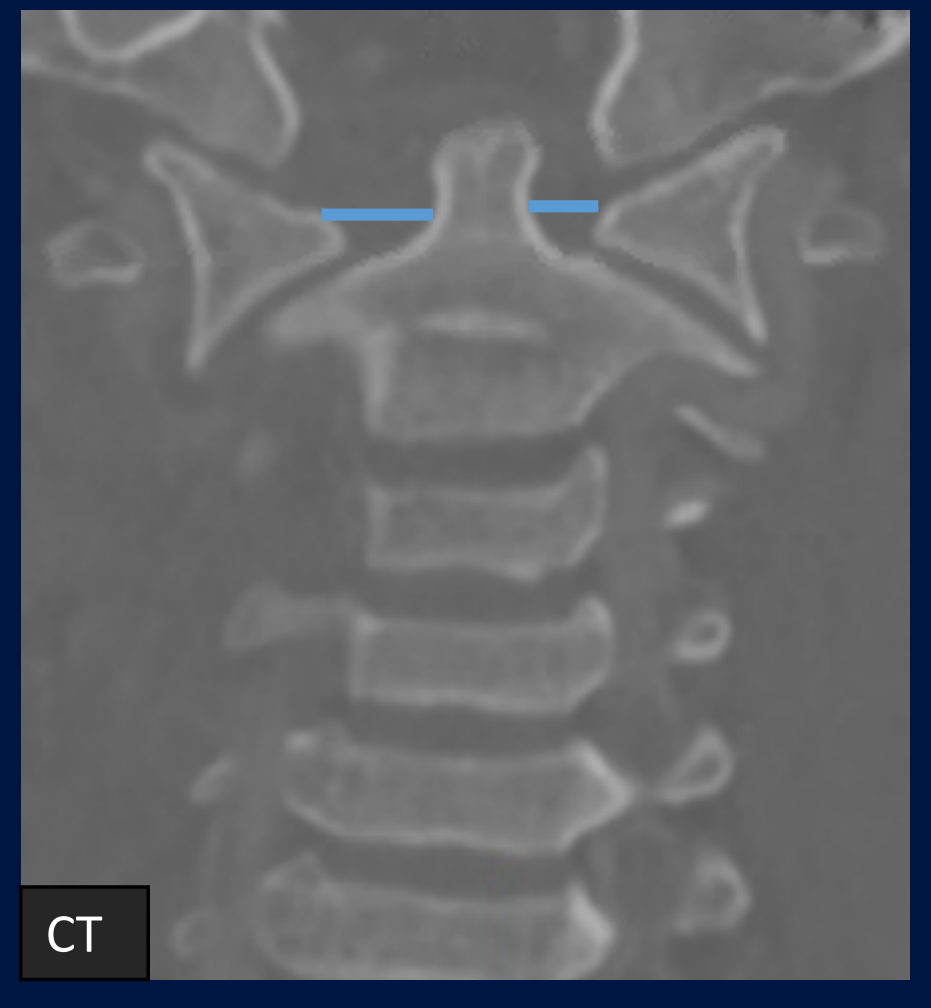

Grisel syndrome refers to non traumatic rotatory atlantoaxial subluxation usually associated with respiratory infection or regional surgery. 3D reformat techniques of PACS, may be utilized to correct for positional errors. Ligamentous laxity causes C1-C2 instability, leading to various types of atlantoaxial subluxation. 
What is the most common bacteria to cause pyogenic osteomyelitis/discitis in children? Click on the box to choose.

V Staphylococcus aureus

\ Streptococcus pyogenes

$\mathbf{X}$ Salmonella osteomyelitis

\ Bartonella henslei

凶 Kingella Kingei 
What is the most common bacteria to cause pyogenic osteomyelitis/discitis in children? Click on the box to choose.

V Staphylococcus aureus

\ Streptococcus pyogenes

$\mathbf{X}$ Salmonella osteomyelitis

\ Bartonella henslei

凶 Kingella Kingei 


\section{Pyogenic spondylitis/ discitis overview}

- Clinical Profile:

- Peak age range: 6 months to 4 years

- Clinical symptoms variable \& nonspecific in children

- Difficulty walking, back/hip pain, fever $(<50 \%)$, irritability

- Inflammatory markers may not be elevated initially including ESR, CRP and WBC

- Site of involvement: Lumbosacral $(75 \%)>$ thoracic $>$ cervical spine

- Pathology: Hematogenous seeding more common than contiguous spread or direct inoculation

- Starts at disc plate with formation of micro-abscesses

- Intervertebral disc \& vertebral growth plates highly vascularized in young children

- Earliest MRI signs: Discitis is seen as hyperintense signal in intervertebral disc, loss of intervertebral disc height and endplate irregularity.

- Main stay of management is IV antibiotics. CT-guided or open biopsy may be indicated if blood cultures negative $\&$ conservative treatment fails. 


\section{Case 4}

- 16 yr. old male presented with mid back pain for few months.

- He had low grade fevers, no increase in white cell count and raised ESR of $80 \mathrm{~mm} / \mathrm{hr}$.

- Images from his initial MRI are as follows: 


\section{Tuberculous spondylodiscitis}
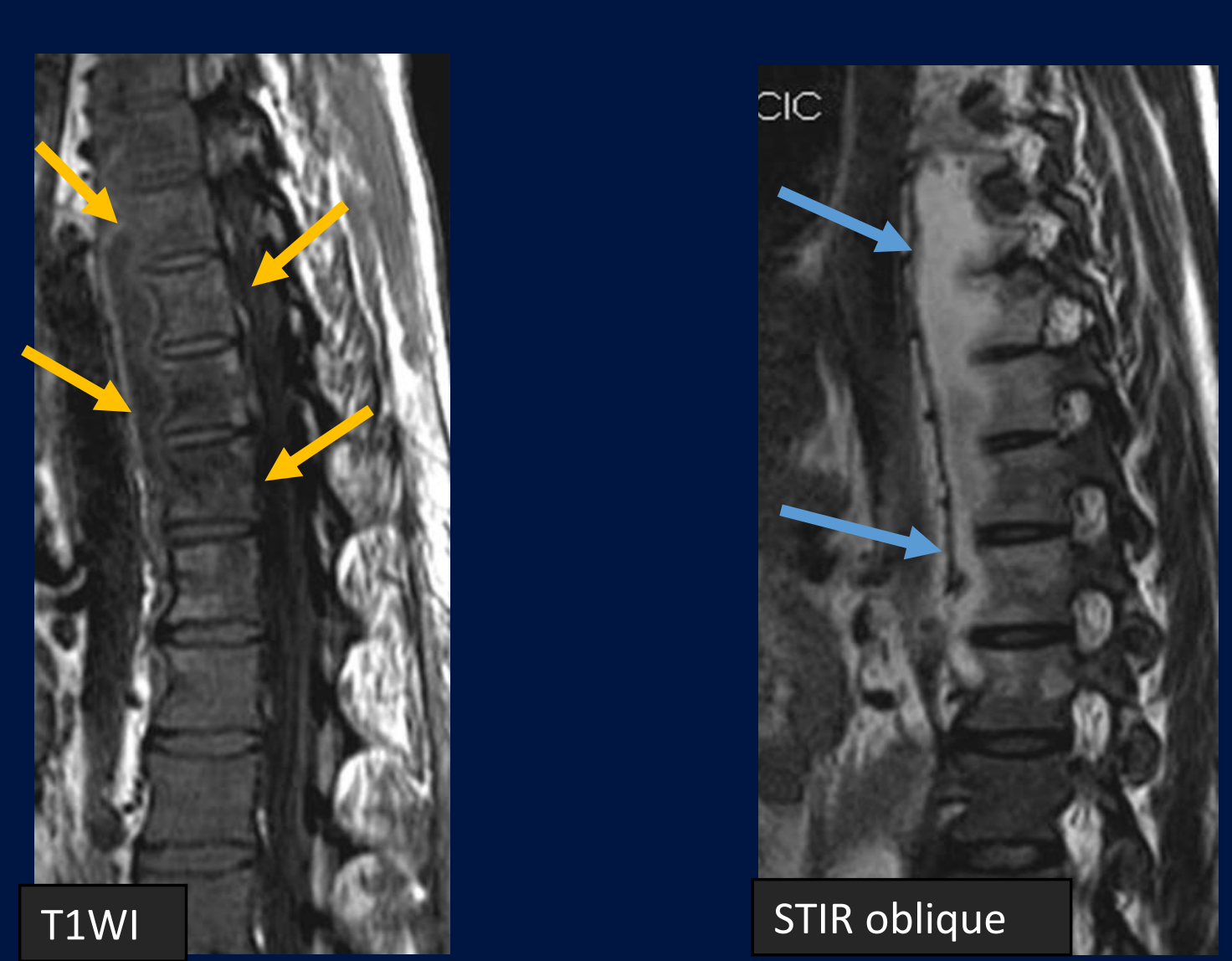

There is abnormal marrow signal involving multiple vertebral bodies best appreciated on T1WI, suggestive of spondylitis. The intervertebral discs are relatively spared. There is prevertebral phlegmonous collection with a subligamentous spread pattern. The patient was unable to afford intravenous contrast, so it was not given. This is a case of tuberculous spondylitis from India. Diagnosis was made on the basis of endemicity of the region, clinical symptoms, raised ESR and imaging findings. He was started on antituberculous treatment and was lost to follow up. 
- Clinical Profile:

- Spinal TB more aggressive in children. Kyphosis and cord compression more common.

- Most common signs/symptoms is chronic back pain ( 95\%) and focal tenderness. Fever is relatively infrequent .

- Lab markers usually show $\uparrow$ ESR.

- Site of involvement:

- Midthoracic or thoracolumbar > lumbar, cervical

- Pathology:

- Spinal TB accounts for $2 \%$ of all TB cases. Concomitant pulmonary TB in $10 \%$ of patients

- Hematogenous or lymphatic spread of granulomatous infection from pulmonary origin

- Initial inoculum in anterior vertebral body

- Spread to noncontiguous vertebral bodies beneath longitudinal ligaments

- Sparing of intervertebral disc secondary to lack of proteolytic enzymes

- Earliest MRI signs:

- Abnormal marrow signal involving multiple vertebral bodies

- Intervertebral discs are relatively spared in early infection

- Large dissecting paraspinal abscesses over considerable distance

- Progressive infection may result in vertebral collapse and gibbus deformity

- Main stay of management :

- Long-term antituberculous medication for at least 1 year

- Surgical decompression in setting of neurologic deficits \pm spinal deformity 


\section{Early signs of infectious spondylodiscitis:}

- Look for very subtle signal abnormalities in:

- Vertebral endplates

- Marrow

- Paraspinal tissues

- Suggest clinical / laboratory correlation and follow up imaging if spinal infection is a possibility. 


\section{References:}

- Khoury, Nabil J., et al. "Imaging of back pain in children and adolescents." Current problems in diagnostic radiology 35.6 (2006): 224244.

- Rossi, Andrea. "Pediatric spinal infection and inflammation." Neuroimaging Clinics 25.2 (2015): 173-191.

- Principi, Nicola, and Susanna Esposito. "Infectious discitis and spondylodiscitis in children." International journal of molecular sciences 17.4 (2016): 539.

- Offiah, A. C. "Acute osteomyelitis, septic arthritis and discitis: differences between neonates and older children." European journal of radiology 60.2 (2006): 221-232.

- Dartnell, J., M. Ramachandran, and M. Katchburian. "Haematogenous acute and subacute paediatric osteomyelitis: a systematic review of the literature." The Journal of bone and joint surgery. British volume 94.5 (2012): 584-595.

- Fernandez, Marisol, Clark L. Carrol, and Carol J. Baker. "Discitis and vertebral osteomyelitis in children: an 18-year review." Pediatrics 105.6 (2000): 1299-1304.

- Chandrasenan, J, et al. "Spondylodiscitis in children: a retrospective series." The Journal of bone and joint surgery. British volume 93.8 (2011): 1122-1125.

- Wiley, A. M., and Josep Trueta. "The vascular anatomy of the spine and its relationship to pyogenic vertebral osteomyelitis." The Journal of bone and joint surgery. British volume 41.4 (1959): 796-809. E VASCULAR ANATOMY OF THE SPINE AND ITS RELATIONSHIP

- Das, Soumyajit, Suvamoy Chakraborty, and Subhajit Das. "Grisel Syndrome in Otolaryngology: A Case Series with Literature Review." Indian Journal of Otolaryngology and Head \& Neck Surgery (2019): 1-4.

- Scheuerman, Oded, et al. "Cervical discitis in children." The Pediatric infectious disease journal 34.7 (2015): 794-795.

- Falip, Céline, et al. "Chronic recurrent multifocal osteomyelitis (CRMO): a longitudinal case series review." Pediatric radiology 43.3 (2013): 355-375.

- Elder, B., \& Wolinsky, J. (2018-11). Basilar Invagination and Cranial Settling. In Spinal Neurosurgery. Oxford, UK: Oxford University Press. Retrieved 24 Mar. 2020, from https://oxfordmedicine.com/view/10.1093/med/9780190887773.001.0001/med9780190887773 -chapter-6.

- Stat Dx 